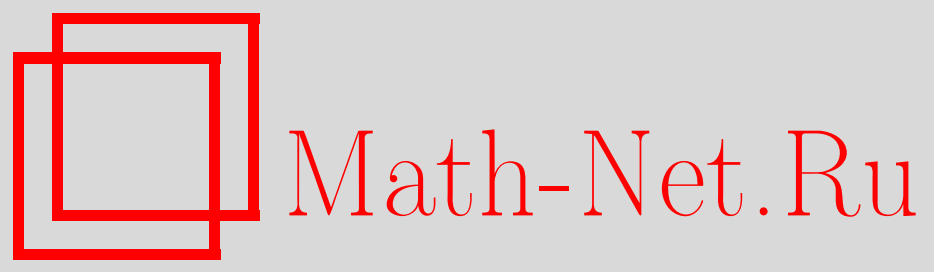

В. В. Дубровский, Регуляризованные следы несамосопряженных операторов, Матем. заметки, 1999, том 65, выпуск 5, 783-787

DOI: https://doi.org/10.4213/mzm1110

Использование Общероссийского математического портала Math-Net.Ru подразумевает, что вы прочитали и согласны с пользовательским соглашением http://www.mathnet.ru/rus/agreement

Параметры загрузки:

IP : 54.157 .27 .8

26 апреля 2023 г., 13:57:26 


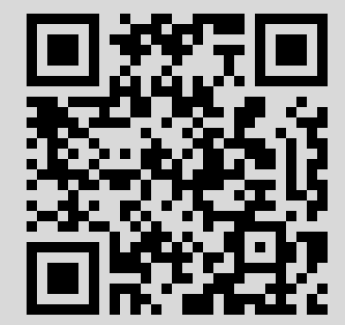




\section{РЕГУЛЯРИЗОВАННЫЕ СЛЕДЫ НЕСАМОСОПРЯЖЕННЫХ ОПЕРАТОРОВ}

\section{В. В. Дубровский}

Проблема вычисления регуляризованных следов восходит к работе И. М. Гельфанда и Б. М. Левитана [1]. Ими был рассмотрен оператор Штурма-Лиувилля с краевыми условиями Дирихле:

$$
\begin{gathered}
-y^{\prime \prime}+q(x) y=\lambda y, \quad 0 \leqslant x \leqslant \pi, \\
y(0)=y(\pi)=0 .
\end{gathered}
$$

Асимптотика упорядоченных по возрастанию собственных чисел $\lambda_{n}$ этой задачи имеет вид

$$
\lambda_{n}=n^{2}+c_{0}+O\left(\frac{1}{n^{2}}\right), \quad c_{0}=\frac{1}{\pi} \int_{0}^{\pi} q(x) d x
$$

поэтому ряд

$$
\sum_{n=1}^{\infty}\left(\lambda_{n}-n^{2}-c_{0}\right)
$$

сходится. Гельфандом и Левитаном было доказано, что

$$
\sum_{n=1}^{\infty}\left(\lambda_{n}-n^{2}-c_{0}\right)=\frac{1}{2} c_{0}-\frac{q(0)+q(\pi)}{4}
$$

где $q(x)$ - дважды дифференцируемая на отрезке $[0, \pi]$ функция.

Л.А. Дикий в работе [2] вычислил регуляризованные следы регулярного оператора ШтурмаЛиувилля высших порядков и исследовал дзета-функцию этого оператора. В работах [3], [4] В. Б. Лидского и В. А. Садовничего вычислены регуляризованные следы всех операторов для обыкновенных дифференциальных операторов со сложным вхождением спектрального параметра.

Для дифференциальных операторов с частными производными высоких порядков впервые была вычислена регуляризованная сумма в работе [5]. На данном этапе развития теории регуляризованных следов следует особое внимание обратить на получение формул регуляризованных следов для дифференциальных операторов с частными производньми второго порядка (см. [6]).

Наша работа посвящена абстрактной формуле Гельфанда-Левитана для несамосопряженных операторов.

Пусть $H$ - сепарабельное комплексное гильбертово пространство, $T$ - полуограниченньй, самосопряженный оператор в $H$ с ядерной резольентой, $\lambda_{n}$ - собственные числа оператора $T$, занумерованные в порядке возрастания их величин с учетом кратности, $v_{n}$ - собственные ортонормированные векторы оператора $T$, отвечаюшие собственным числам $\lambda_{n}$. Далее, $P$ - ограниченный,

Автор поддержан грантом 192d общеобразовательного фонда Сороса.

(C) В. В. Дувровский 
вообще говоря, несамосопряженный оператор в $H, \mu_{n}$ - собственные числа оператора $T+P$, занумерованные в порядке возрастания их действительных частей с учетом алгебраической кратности. Предположим, что $\lambda_{n} \sim c n^{\alpha}, c>0, \alpha>1$.

Очевидны следующие утверждения (см. [7], [8]).

Лемма 1. Существует бесконечная последовательность натуральных чисел $n_{m}$ такая, чmo

$$
\lambda_{n_{m+1}}-\lambda_{n_{m}} \geqslant \frac{c}{2} n_{m}^{\alpha-1}>0 .
$$

ЛЕмма 2. Если $\left|n-n_{m}\right| \geqslant c_{0} n_{m}$, әде $c_{0}$ - фиксированное, сколь угодно малое, положительное число, то

$$
\left|\lambda_{n}-\lambda_{n_{m}}\right| \geqslant \operatorname{const}\left\{n_{m}^{\alpha}, n^{\alpha}\right\}>0 .
$$

Оценим сверху ядерную норму резольвенты оператора $T$.

ЛЕмма 3. На окружностях $\gamma_{n_{m}}=\left\{\lambda \mid \lambda=r_{n_{m}}=\left(\lambda_{n_{m}}+\lambda_{n_{m+1}}\right) / 2\right\}$ справедлива оченка сверху ядерной нормы резольвенты оператора $T$ :

$$
\left\|(T-\lambda E)^{-1}\right\|_{1} \leqslant \text { const } n_{m}^{-\alpha+2}
$$

əде $\lambda \in \gamma_{n_{m}}$ u const не зависит от $n_{m}$.

ДокАЗАТЕЛЬСтво. Имеем при $\lambda \in \gamma_{n_{m}}$

$$
\begin{aligned}
\left\|(T-\lambda E)^{-1}\right\|_{1} & \equiv \sum_{n=1}^{\infty}\left|\lambda-\lambda_{n}\right|^{-1}=\sum_{n=1}^{n_{m}-\left[c_{0} n_{m}\right]}+\sum_{n=n_{m}-\left[c_{0} n_{m}\right]+1}^{n_{m}+\left[c_{0} n_{m}\right]}+\sum_{n=n_{m}+\left[c_{0} n_{m}\right]+1}^{\infty} \\
& =J_{1}+J_{2}+J_{3} .
\end{aligned}
$$

Оценим сверху каждое из слагаемых $J_{j}, j=1,2,3$. По лемме 2 получаем, что

$$
J_{1}=\sum_{n=1}^{n_{m}-\left[c_{0} n_{m}\right]}\left|\lambda-\lambda_{n}\right|^{-1} \leqslant \sum_{n=1}^{n_{m}-\left[c_{0} n_{m}\right]} \frac{\text { const }}{n^{\alpha}} \leqslant \text { const } n_{m}^{-\alpha+1} .
$$

По лемме 1 имеем

$$
J_{2} \leqslant \frac{2 c_{0} n_{m}}{n_{m}^{\alpha-1}}=\text { const } n_{m}^{-\alpha+2} .
$$

$J_{3}$ оценивается так же, как и $J_{1}$, с помощью леммы 2 . Лемма 3 доказана.

Далее, будем пользоваться методикой работы [9] для изучения функции

$$
f(\lambda)=\operatorname{Sp} \lambda(T+P-\lambda E)^{-1}-\operatorname{Sp} \lambda(T-\lambda E)^{-1}+\operatorname{Sp} \lambda(T-\lambda E)^{-1} P(T-\lambda E)^{-1} .
$$

ЛЕмма 4. При $\alpha>1$ существует и конечен предел

$$
\lim _{m \rightarrow \infty} \sum_{n=1}^{n_{m}}\left\{\mu_{n}-\lambda_{n}-\left(P v_{n}, v_{n}\right)\right\}
$$


ДоКАЗАТЕЛЬСтво. Сначала с помощью леммы 3 оценим сверху $|f(\lambda)|$ на окружностях $\gamma_{n_{m}}$ :

$$
\begin{aligned}
\sup _{\lambda \in \gamma_{n}}|f(\lambda)| r_{n_{m}}^{-2}= & \sup _{\lambda \in \gamma_{n_{m}}}\left\{\lambda \operatorname{Sp}(T+P-\lambda E)^{-1}-\lambda \operatorname{Sp}(T-\lambda E)^{-1}\right. \\
& \left.-\lambda \operatorname{Sp}(T-\lambda E)^{-1} P(T-\lambda E)^{-1}\right\} r_{n_{m}}^{-2} \\
\leqslant & \sup _{\lambda \in \gamma_{n_{m}}}\left\{|\lambda|\left\|(T+P-\lambda E)^{-1}\right\|_{1}+|\lambda|\left\|(T-\lambda E)^{-1}\right\|_{1}\right. \\
& \left.+|\lambda|\left\|(T-\lambda E)^{-1}\right\|_{1}\|P\|_{1}\left\|(T-\lambda E)^{-1}\right\|_{1}\right\} r_{n_{m}}^{-2} \\
\leqslant & \operatorname{const}\left\{\frac{n_{m}^{\alpha} n_{m}^{-\alpha+2}}{n_{m}^{2 \alpha}}+\frac{n_{m}^{\alpha} n_{m}^{-\alpha+2}}{n_{m}^{2 \alpha}}+\frac{n_{m}^{\alpha} n_{m}^{-\alpha+2} n_{m}^{-\alpha+1}}{n_{m}^{2 \alpha}}\right\} \\
\leqslant & \frac{\text { const }}{n_{m}^{2 \alpha-2}} \rightarrow 0, \quad \alpha>1 .
\end{aligned}
$$

Обозначим совокупность собственных чисел операторов $T+P$ и $T$ через $\left\{\beta_{n}\right\}_{n=0}^{\infty}$, занумерованных в порядке возрастания их модулей с учетом кратности, причем $\beta_{0}=0$.

Представим

$$
\frac{1}{\lambda-z}=\frac{1}{\lambda} \frac{1}{\left(1-\frac{z}{\lambda}\right)}=\frac{1}{\lambda}+\frac{z}{\lambda^{2}}+\frac{z^{2}}{\lambda^{3}}+\frac{z^{3}}{\lambda^{4}}+\frac{1}{(\lambda-z)} \frac{z^{4}}{\lambda^{4}}
$$

Тогда вблизи $\gamma_{n_{m}}$

$$
f(z)=\sum_{0}^{2 n_{m}} G_{n}(z)+\sum_{j=0}^{2} \frac{z^{j}}{2 \pi i} \int_{\gamma_{n_{m}}} \frac{f(\lambda)}{\lambda^{1+j}} d \lambda+\frac{1}{2 \pi i} \int_{\gamma_{n_{m}}} \frac{f(\lambda)}{\lambda-z} \frac{z^{3}}{\lambda^{3}} d \lambda .
$$

Далее,

$$
\underset{\lambda=\beta_{n}}{\operatorname{res}} \frac{f(\lambda)}{\lambda-z}=-G_{n}(\lambda), \quad n=0, \ldots, 2 n_{m}
$$

Если

то (см. [9])

$$
f(\lambda)=\frac{A_{-j_{k}}^{(n)}}{\left(\lambda-\beta_{n}\right)^{\gamma_{k}}}+\cdots+\frac{A_{-1}^{(n)}}{\lambda-\beta_{n}}+A_{0}^{(n)}+A_{1}^{(n)}\left(\lambda-\beta_{n}\right)+\cdots
$$

$$
G_{n}(\lambda)=\frac{A_{-j_{k}}^{(n)}}{\left(\lambda-\beta_{n}\right)^{\gamma_{k}}}+\cdots+\frac{A_{-1}^{(n)}}{\lambda-\beta_{n}}
$$

Суммирование в формуле (1) справа в первом выражении идет без учета кратности величин $\beta_{k}$. Тогда

$$
\left|\frac{1}{2 \pi i} \int_{\gamma_{n_{m}}} \frac{f(\lambda)}{\lambda-z} \frac{z^{4}}{\lambda^{4}} d \lambda\right| r_{n_{m}} \leqslant \text { const } \frac{2 \pi n_{m}^{\alpha}|z|^{4}}{n_{m}^{4 \alpha} n_{m}^{\alpha-1}} n_{m}^{\alpha} n_{m}^{-\alpha+2}=\frac{\text { const }}{n_{m}^{4 \alpha-3}} \rightarrow 0 .
$$

Отсюда

$$
f(z)=\sum_{0}^{2 n_{m}} G_{k}(z)+\sum_{j=0}^{3} \frac{z^{j}}{2 \pi i} \int_{\gamma_{n_{m}}} \frac{f(\lambda)}{\lambda^{1+j}} d \lambda+O\left(\frac{1}{n_{m}^{4 \alpha-3}}\right) .
$$

Проинтегрируем функцию $f(z)$ по окружности $\gamma_{n_{m}}$ с множителем $-1 /(2 \pi i)$ по $d z$ :

$$
\begin{aligned}
-\frac{1}{2 \pi i} \oint_{\gamma_{n}} f(\lambda) d \lambda= & \operatorname{Sp}-\frac{1}{2 \pi i} \oint_{\gamma_{n_{m}}} \lambda \operatorname{Sp}(T+P-\lambda E)^{-1} d \lambda \\
& +\frac{1}{2 \pi i} \oint_{\gamma_{n_{m}}} \lambda \operatorname{Sp}(T-\lambda E)^{-1} d \lambda \\
& -\frac{1}{2 \pi i} \oint_{\gamma_{n_{m}}} \lambda \operatorname{Sp}(T-\lambda E)^{-1} P(T-\lambda E)^{-1} d \lambda+\oint_{\gamma_{n_{m}}} \frac{O(1)}{n_{m}^{4 \alpha-3}} d \lambda \\
= & \sum_{n=1}^{n_{m}}\left[\mu_{n}-\lambda_{n}-\left(P v_{n}, v_{n}\right)\right]+\frac{O(1)}{n_{m}^{3 \alpha-3}} .
\end{aligned}
$$


Следовательно, предел в формулировке леммы 4 существует и конечен. Лемма 4 доказана.

Tеорема 5. Eсли $\alpha>1$, mo

$$
\lim _{m \rightarrow \infty} \sum_{n=1}^{n_{m}}\left[\mu_{n}-\lambda_{n}-\left(P v_{n}, v_{n}\right)\right]=0 .
$$

ДоКАЗАТЕЛЬСтво. Имеем

$$
\begin{aligned}
\mid \lim _{m \rightarrow \infty} & \sum_{n=1}^{n_{m}}\left[\mu_{n}-\lambda_{n}-\left(P v_{n}, v_{n}\right)\right] \mid \\
= & \mid \lim _{\lambda \rightarrow+\infty} \lambda^{2}\left\{-\operatorname{Sp}(T+P-\lambda E)^{-1}+\operatorname{Sp}(T-\lambda E)^{-1}\right. \\
& \left.-\sum_{n} \frac{\left(P v_{n}, v_{n}\right)}{\left(\lambda_{n}+\lambda\right)\left(\lambda+\mu_{n}\right)}\right\} \mid \\
= & \left|\lim _{\lambda \rightarrow+\infty} \lambda^{2}\left\{-\operatorname{Sp}(T+P-\lambda E)^{-1}+\operatorname{Sp}(T-\lambda E)^{-1}-\sum_{n} \frac{\left(P v_{n}, v_{n}\right)}{\left(\lambda_{n}+\lambda\right)^{2}}\right\}\right| \\
= & \left|\lim _{\lambda \rightarrow+\infty} \lambda^{2}\right|-\operatorname{Sp}(T+P-\lambda E)^{-1}+\operatorname{Sp}(T-\lambda E)^{-1} \\
& -\operatorname{Sp}(T-\lambda E)^{-1} P(T-\lambda E)^{-1}|| \\
= & \left|\lim _{\lambda \rightarrow+\infty} \lambda^{2} \operatorname{Sp}\left\{(T+P-\lambda E)^{-1} P(T-\lambda E)^{-1} P(T-\lambda E)^{-1}\right\}\right| \\
\leqslant & \lim _{\lambda \rightarrow+\infty} \lambda^{2} \frac{\operatorname{const}}{\lambda^{2}}\left\|(T-\lambda E)^{-1}\right\|_{1}=0,
\end{aligned}
$$

так как $\lim _{\lambda \rightarrow \infty}\left\|(T-\lambda E)^{-1}\right\|_{1}=0$.

Пусть $T_{0}=-\Delta$ - оператор Лапласа, заданный на прямоугольнике $\Pi=\{(x, y) \mid 0<x<$ $a, 0<y<b\}, P$ - оператор умножения на, вообще говоря, комплексную дважды непрерьвно дифференцируемую функцию $p(x, y), \lambda_{t}^{\alpha}-$ собственные числа оператора $T=T_{0}^{\alpha}$, занумерованные в порядке возрастания их величин с учетом кратности, $t=1, \ldots, \infty$. Из элементарных соображений известно, что

$$
\begin{aligned}
& \lambda_{t}=\lambda_{m_{t}, n_{t}}=\frac{\pi^{2}}{a^{2}} m_{t}^{2}+\frac{\pi^{2}}{b^{2}} n_{t}^{2}, \quad m_{t}, n_{t} \in \mathbb{N}, \\
& v_{t}=v_{m_{t}, n_{t}}=\frac{2}{\sqrt{a b}} \sin \frac{\pi}{a} m_{t} x \sin \frac{\pi}{b} n_{t} y,
\end{aligned}
$$

где $T_{0}^{\alpha}=\int \lambda^{\alpha} d E(\lambda)$, a $E(\lambda)$ - спектральное разложение единицы оператора $T_{0}, \alpha>1$. Тогда

$$
\lambda_{t}^{\alpha} \sim c_{\alpha} t^{\alpha}
$$

$\mu_{m, n}^{\alpha}$ - собственные числа оператора $T_{0}^{\alpha}+P$, занумерованные в порядке возрастания их действительных частей с учетом кратности. Тогда $\left|\mu_{m, n}^{\alpha}-\lambda_{m, n}^{\alpha}\right| \leqslant$ const.

Из работы [10] вытекает, что справедлива следующая формула первого регуляризованного следа для оператора $T_{0}^{\alpha}+P$ при $\alpha>1$

$$
\begin{aligned}
\sum_{n>0, m>0} & \left(\left(\mu_{m, n}^{\alpha}-\left(\frac{\pi^{2}}{a^{2}} m^{2}+\frac{\pi^{2}}{b^{2}} n^{2}\right)\right)\right. \\
& \left.-\frac{1}{a b} \int_{0}^{a} \int_{0}^{b} p(x, y)\left(1-\cos \frac{2 \pi}{a} \cos x-\cos \frac{2 \pi}{b} \cos y\right) d x d y\right) \\
= & \frac{p(0,0)+p(0, b)+p(a, 0)+p(a, b)}{16}+\frac{1}{8 b} \int_{0}^{b}(p(a, y)+p(0, y)) d y \\
& +\frac{1}{8 a} \int_{0}^{a}(p(x, b)+p(x, 0)) d x-\frac{1}{4 a b} \int_{0}^{a} \int_{0}^{b} p(x, y) d x d y
\end{aligned}
$$


Суммирование в этой формуле слева производится со скобками. Скобки можно снять, если $a^{2} / b^{2}$ - рациональное число.

Автор благодарит В.Е. Подольского за участие в обсуждении работы.

\section{СПИСОК ЦИТИРОВАННОЙ ЛИТЕРАТУРЫ}

1. Гельфанд И. М., Левитан Б. М. // Докл. АН СССР. 1953. Т. 88. № 4. С. 591-596. 2. Дикий Л. А. // Изв. АН СССР. Сер. матем. 1955. Т. 18. №4. С. 187-200. 3. Лидский В. Б., Садовничий В. А. // Докл. АН СССР. 1967. Т. 176. № 2. С. 259-262. 4. Лидский В. Б., Садовничий В.А.// Функцион. анализ и его прилож. 1967. Т. 1. № 2. С. 52-59. 5. Садовничий В. А., Дубровский В. В. // Дифференц. уравнения. 1977. Т. 13. №11. С. 2033-2042. 6. Садовничий В.А., Дубровский В.В. // Докл. АН СССР. 1991. Т. 319. №1. С. 61-62. 7. Дубровский В. В., Печенцов А. С. // Дифференц. уравнения. 1993. Т. 299. № 5. С. 852-868. 8. Дубровский В.В. // УМН. 1991. Т. 46. №3. С. 187-188. 9. Маркушевич А. И. Краткий курс теории аналитических функций. М.: Наука, 1966. 10. Дубровский В. В. // Труды семинара им. И. Г. Петровского. 1978. Т. 4. С. 227-231.

Магнитогорский государственный педагогический институт

Поступило 30.09.1996

Исправленный вариант

30.12 .1997 